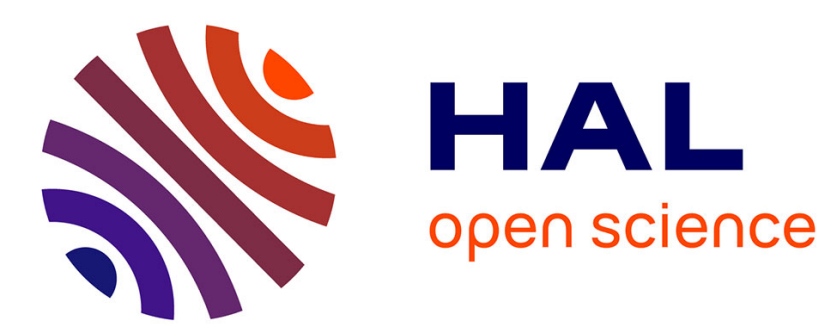

\title{
On well-posedness of scattering problems in a Kirchhoff-Love infinite plate
}

Laurent Bourgeois, Christophe Hazard

\section{To cite this version:}

Laurent Bourgeois, Christophe Hazard. On well-posedness of scattering problems in a Kirchhoff-Love infinite plate. SIAM Journal on Applied Mathematics, 2020, 80 (3), pp.1546-1566. hal-02334004

\section{HAL Id: hal-02334004 \\ https://hal-ensta-paris.archives-ouvertes.fr/hal-02334004}

Submitted on 25 Oct 2019

HAL is a multi-disciplinary open access archive for the deposit and dissemination of scientific research documents, whether they are published or not. The documents may come from teaching and research institutions in France or abroad, or from public or private research centers.
L'archive ouverte pluridisciplinaire HAL, est destinée au dépôt et à la diffusion de documents scientifiques de niveau recherche, publiés ou non, émanant des établissements d'enseignement et de recherche français ou étrangers, des laboratoires publics ou privés. 


\title{
On well-posedness of scattering problems in a Kirchhoff-Love infinite plate
}

\author{
Laurent Bourgeois* and Christophe Hazard*
}

October 25, 2019

\begin{abstract}
We address scattering problems for impenetrable obstacles in an infinite elastic Kirchhoff-Love two-dimensional plate. The analysis is restricted to the purely bending case and the time-harmonic regime. Considering four types of boundary conditions on the obstacle, well-posedness for those problems is proved with the help of a variational approach: (i) for any wave number $k$ when the plate is clamped, simply supported or roller supported; (ii) for any $k$ except a discrete set when the plate is free (this set is finite for convex obstacles).
\end{abstract}

\section{Introduction}

We consider the diffraction of an incident wave by an impenetrable obstacle within an infinite elastic thin plate characterized by the Kirchhoff-Love model. More precisely, we focus on the pure bending case in the frequency domain. Such an impenetrable obstacle $\mathcal{D} \subset \mathbb{R}^{2}$ can be modeled by a bounded open domain of class $C^{3}$ (see Remark 2). Denoting $\Omega:=\mathbb{R}^{2} \backslash \overline{\mathcal{D}}$, the scattered field $v^{\mathrm{s}}$ satisfies the problem

$$
\begin{cases}\Delta^{2} v^{\mathrm{s}}-k^{4} v^{\mathrm{s}}=0 & \text { in } \Omega, \\ \left(\mathcal{B}_{1}\left(v^{\mathrm{s}}+u^{\mathrm{i}}\right), \mathcal{B}_{2}\left(v^{\mathrm{s}}+u^{\mathrm{i}}\right)\right)=(0,0) & \text { on } \partial \Omega, \\ \lim _{r \rightarrow+\infty} \int_{\partial B_{r}}\left|\frac{\partial v^{\mathrm{s}}}{\partial n}-\mathrm{i} k v^{\mathrm{s}}\right|^{2} \mathrm{~d} s=0, & \end{cases}
$$

where $k>0$ is the wave number, $u^{\mathrm{i}}$ is an incident field which satisfies $\Delta^{2} u^{\mathrm{i}}-k^{4} u^{\mathrm{i}}=0$ in $\mathbb{R}^{2}, B_{r}$ is the open ball centered at 0 and of radius $r$ while $n$ is the outward normal to $B_{r}$ and $s$ is the curvilinear measure on $\partial B_{r}$.

The first equation of (1) comes from the equation of the flexural waves in a plate

$$
D \Delta^{2} u+\rho h \frac{\partial^{2} u}{\partial t^{2}}=0
$$

with $D:=E h^{3} / 12\left(1-\nu^{2}\right)$, where $E>0$ is the Young's modulus, $\nu \in[0,1 / 2)$ is the Poisson's ratio, $h$ is the thickness and $\rho$ is the density per unit of volume. In the time-harmonic case at frequency $\omega$, the equation (2) coincides with the first equation of (1) with $k$ defined by $k^{4}=\rho h \omega^{2} / D$. We mention that several plate models in the time domain, in particular the one given by (2), are derived in [13] starting from a three dimensional setting. Such models enable us to reduce the spatial dimension of the elasticity problem from 3 to 2 provided the thickness $h$ is small with respect to the wavelength $2 \pi / k$.

The surface differential operators $\mathcal{B}_{1}, \mathcal{B}_{2}$ involved in the second line of (1) characterize the boundary conditions on $\partial \Omega$. In order to specify these operators, let us introduce some notations. In the sequel, a

\footnotetext{
*POEMS ${ }^{1}$, ENSTA-ParisTech, 828 boulevard des Maréchaux, $91120 \quad$ Palaiseau, France (christophe.hazard@ensta-paristech.fr, sandrine.paolantoni@ensta-paristech.fr).

${ }^{1}$ POEMS (Propagation d'Ondes: Etude Mathématique et Simulation) is a mixed research team (UMR 7231) between CNRS (Centre National de la Recherche Scientifique), ENSTA-Paris (Ecole Nationale Supérieure de Techniques Avancées) and INRIA (Institut National de Recherche en Informatique et en Automatique).
} 
generic point of $\mathbb{R}^{2}$ is denoted $x=\left(x_{1}, x_{2}\right)$. Moreover, $n=\left(n_{1}, n_{2}\right)$ denotes the unit normal vector to $\partial \Omega$ directed into the exterior of $\Omega$ (that is, the interior of $\mathcal{D})$, while $t$ is the unit tangent vector such that $t$ is obtained from $n$ by a $\pi / 2$ counterclockwise rotation (in other words, $t_{1}=-n_{2}$ and $t_{2}=n_{1}$ ). The curvilinear abscissa associated with vector $t$ is denoted $s$ : it defines the curvilinear measure d $s$ used in boundary integrals. We also define the surface differential operators

$$
\frac{\partial}{\partial n}:=n_{1} \frac{\partial}{\partial x_{1}}+n_{2} \frac{\partial}{\partial x_{2}} \quad \text { and } \quad \frac{\partial}{\partial s}:=-n_{2} \frac{\partial}{\partial x_{1}}+n_{1} \frac{\partial}{\partial x_{2}} .
$$

The operators $\mathcal{B}_{1}$ and $\mathcal{B}_{2}$ are two among the following four surface differential operators: $I$ (trace operator), $\partial / \partial n, M$ and $N$, where $M$ and $N$ are defined as follows:

$$
\left\{\begin{aligned}
M u & :=\nu \Delta u+(1-\nu) M_{0} u \\
N u & :=-\frac{\partial}{\partial n} \Delta u-(1-\nu) \frac{\partial}{\partial s} N_{0} s
\end{aligned}\right.
$$

where $M_{0}$ and $N_{0}$ are given by

$$
\left\{\begin{aligned}
M_{0} u & :=\frac{\partial^{2} u}{\partial x_{1}^{2}} n_{1}^{2}+2 \frac{\partial^{2} u}{\partial x_{1} \partial x_{2}} n_{1} n_{2}+\frac{\partial^{2} u}{\partial x_{2}^{2}} n_{2}^{2} \\
N_{0} u & :=\frac{\partial^{2} u}{\partial x_{1} \partial x_{2}}\left(n_{1}^{2}-n_{2}^{2}\right)-\left(\frac{\partial^{2} u}{\partial x_{1}^{2}}-\frac{\partial^{2} u}{\partial x_{2}^{2}}\right) n_{1} n_{2} .
\end{aligned}\right.
$$

Some alternative useful expressions of $M u$ and $N u$ are

$$
\left\{\begin{aligned}
M u & =\Delta u-(1-\nu)\left(\frac{\partial^{2} u}{\partial s^{2}}+\gamma \frac{\partial u}{\partial n}\right) \\
N u & =-\frac{\partial}{\partial n} \Delta u-(1-\nu) \frac{\partial}{\partial s}\left(\frac{\partial^{2} u}{\partial s \partial n}-\gamma \frac{\partial u}{\partial s}\right)
\end{aligned}\right.
$$

where $\gamma(s)$ is the curvature defined by $\partial n / \partial s=\gamma \tau$. It should be noted that physically, $D M$ corresponds to the bending moment while $D N$ corresponds to the transverse force. In what follows, four different cases for the boundary conditions on $\partial \Omega$ will be considered:

1. $\mathcal{B}_{1}=I$ and $\mathcal{B}_{2}=\partial / \partial n$ : clamped plate,

2. $\mathcal{B}_{1}=I$ and $\mathcal{B}_{2}=M$ : simply supported plate,

3. $\mathcal{B}_{1}=\partial / \partial n$ and $\mathcal{B}_{2}=N$ : roller supported plate,

4. $\mathcal{B}_{1}=M$ and $\mathcal{B}_{2}=N$ : free plate.

We emphasize that except the third case, these boundary conditions are classical and relevant from the physical point of view. For instance, the last one models a hole within the plate.

Lastly, the third line of (1) is the radiation condition at infinity. Essentially, it specifies that among the scattered waves, only the outgoing ones are physically acceptable. Such kind of condition is required to guarantee well-posedness of scattering problems in unbounded domains.

The main result of this paper is the following.

Theorem 1. The problem (1) has a unique solution in $H_{\mathrm{loc}}^{2}(\Omega)$

- for any $k>0$ if $\left(\mathcal{B}_{1}, \mathcal{B}_{2}\right)=(I, \partial / \partial n)$ (clamped plate), $\left(\mathcal{B}_{1}, \mathcal{B}_{2}\right)=(I, M)$ (simply supported plate) or $\left(\mathcal{B}_{1}, \mathcal{B}_{2}\right)=(\partial / \partial n, N)$ (roller supported),

- for any $k>0$ except for at most a countable set of numbers $k_{n}, n \in \mathbb{N}$, such that $k_{n} \rightarrow+\infty$, if $\left(\mathcal{B}_{1}, \mathcal{B}_{2}\right)=(M, N)$ (free plate).

Remark 2. Concerning the regularity of the domain $\mathcal{D}$ for which Theorem 1 holds, $\mathcal{D}$ shall be at least of class $C^{2}$ to correctly define the surface operators $M$ and $N$ in (3). Such regularity is enough for Theorem 1 to hold for the clamped, simply supported and roller supported plate. But the strategy we use to prove Theorem 1 for the free plate needs $\mathcal{D}$ to be $C^{3}$. Such assumption might be purely technical. 
The radial case, that is when $\mathcal{D}$ is a disk, was addressed in [14] where a closed-form expression of the solution was obtained. Note that in the present paper, well-posedness in the radial case is proved for any boundary conditions above and for any $k$ (see Theorem 9 when the curvature $\gamma$ is supposed to be constant). To our best knowledge, well-posedness of problem (1) for an obstacle of any shape has never been fully justified. In contrast with plate problems in the static case, which benefit from a huge amount of contributions, plate problems in the dynamic case are less popular. If we restrict ourselves to the mathematical justification of existence and uniqueness for scattering problems in the frequency domain, the number of contributions is very low. However we mention the recent article [19] which in particular addresses existence and uniqueness for solutions of a similar scattering problem involving a biharmonic operator perturbed by zero and first order terms. The proof of well-posedness in [19] is based on a Lippmann-Schwinger integral equation and relies on the assumption that $k$ is sufficiently large. The proof we propose for Theorem 1 is based on a variational approach. As highlighted in the statement of Theorem 1, uniqueness in the case of a free plate for any $k$ is still an open problem for us. Our uniqueness proof for such boundary condition classically relies on the Analytic Fredholm theorem [7, Theorem 8.26], but the latter is applied in an original way to an eigenvalue problem set on the boundary of the obstacle and involving the trace and the normal derivative of the scattered field. As our uniqueness result excludes a possible countable set of frequencies, it is natural to wonder if such exceptional frequencies actually exist, that is if trapped modes can really occur for the free plate. Let us mention some contributions which prove existence of trapped modes in situations which are close to ours, for example the case of a simply supported strip in the presence of a free obstacle [16] or the case of an infinite thick plate in the presence of a free obstacle and $\nu=0$ [9]. It is interesting to note that for a circular hole, the trapped modes disappear when the thickness of such thick plate tends to 0 , in view of Theorem 9 .

Before giving the outline of our article, let us say a few words about the numerical resolution of problem (1). It could be noted that some numerical methods were already used to solve problems of type (1), for instance in [8] with the help of Perfectly Matched Layers or in [17] with the help of a boundary element method. As a by-product of the present paper, by using a Dirichlet-to-Neumann operator we alternatively propose a weak formulation in the bounded domain $\Omega \cap B_{R}$ which is equivalent to the strong problem (1). Such weak formulation can be directly discretized with the help of a finite element method, as done in [4]. Lastly, proving well-posedness for problem (1) paves the way to the corresponding inverse problem: retrieve the obstacle $\mathcal{D}$ given some scattering data. This is the main concern of [4].

The article is organized as follows. The second section can be viewed as a preliminary work on useful properties that are equivalent to the radiation condition given in problem (1). Section 3 is dedicated to a uniqueness proof for problem (1). In section 4, we introduce the so-called Dirichlet problem outside a ball, which is nothing but the particular case of problem (1) when the obstacle $\mathcal{D}$ is a disc $B_{R}$ and the plate is clamped. Existence of a solution is proved for that problem, which implies that the Dirichlet problem outside a ball is well-posed with the help of section 3. This enables us to properly define a Dirichlet-to-Neumann operator on the boundary $\partial B_{R}$. Such operator is used in section 5 to prove that problem (1) is equivalent to a problem set in the bounded domain $\Omega \cap B_{R}$ for which we give a weak formulation. The Fredholm character of this equivalent problem is proved in section 6 . By gathering the results of section 3 and section 6 , we finally complete the existence/uniqueness proof of Theorem 1 .

In order to avoid the appearance of non meaningful constants in inequalities, hereafter we employ the symbols $\lesssim$ and $\gtrsim$ which mean that the inequality is satisfied up to a positive factor which does not depend on the parameters involved in the inequality (for instance, $|f(x)| \lesssim 1$ means that $f$ is bounded).

\section{The radiation condition}

The aim of this section is to show that the radiation condition in problem (1) can be reformulated equivalently as a series expansion of $v^{\mathrm{s}}$ which results from separation of variables in polar coordinates $(r, \theta)$. This expansion is an essential tool in our proof of Theorem 1. It involves the Hankel functions of the first kind $H_{m}^{(1)}$, for $m \in \mathbb{Z}$, (see, e.g. [1]), as well as

$$
\xi_{m}(\theta):=\frac{\mathrm{e}^{\mathrm{i} m \theta}}{\sqrt{2 \pi}} \quad \text { for } \theta \in[0,2 \pi) \text { and } m \in \mathbb{Z} .
$$


Note that if we identify the interval $[0,2 \pi)$ with the unit circle $S_{1}$ in $\mathbb{R}^{2}$, then each function $\xi_{m}$ appears as a unit vector of $L^{2}\left(S_{1}\right)$ (thanks to the factor $1 / \sqrt{2 \pi}$ in (4)). It is actually well-known (see, e.g., [12]) that the family $\left\{\xi_{m} ; m \in \mathbb{Z}\right\}$ is an orthonormal basis of $L^{2}\left(S_{1}\right)$ and for any $s>0$, an orthogonal basis of $H^{s}\left(S_{1}\right)$ for the inner product which derives from the following norm

$$
\|\xi\|_{H^{s}\left(S_{1}\right)}:=\left(\sum_{m \in \mathbb{Z}}\left(1+m^{2}\right)^{s}\left|\left(\xi, \xi_{m}\right)_{L^{2}\left(S_{1}\right)}\right|^{2}\right)^{1 / 2} .
$$

For brevity, we say that a solution $u$ to $\Delta^{2} u-k^{4} u=0$ outside a given ball $B_{R}$ (for some $R>0$ ) is radiating if it satisfies the radiation condition

$$
\lim _{r \rightarrow+\infty} \int_{\partial B_{r}}\left|\frac{\partial u}{\partial n}-\mathrm{i} k u\right|^{2} \mathrm{~d} s=0 .
$$

The two following propositions, which are somehow converse to each other, gather the mains results about such radiating solutions that are needed in the present paper. Their respective proofs use mainly arguments which are well-known in the context of Helmhotz equation (see [7]).

Proposition 3. Suppose that $u$ is a radiating solution to $\Delta^{2} u-k^{4} u=0$ in $\mathbb{R}^{2} \backslash \overline{B_{R}}$ for some $R>0$. Then there exist two families of complex numbers $\alpha_{m}$ and $\beta_{m}$, for $m \in \mathbb{Z}$, such that $u$ can be split into the sum of a propagative part $u^{\mathrm{pr}}$ and an evanescent part $u^{\mathrm{ev}}$ as follows:

$$
u=u^{\mathrm{pr}}+u^{\mathrm{ev}} \text { with }\left\{\begin{aligned}
u^{\mathrm{pr}}(r, \theta) & :=\sum_{m \in \mathbb{Z}} \alpha_{m} H_{m}^{(1)}(k r) \xi_{m}(\theta), \\
u^{\mathrm{ev}}(r, \theta) & :=\sum_{m \in \mathbb{Z}} \beta_{m} H_{m}^{(1)}(\mathrm{i} k r) \xi_{m}(\theta),
\end{aligned}\right.
$$

where both series are absolutely and uniformly convergent on compact subsets of $\mathbb{R}^{2} \backslash \overline{B_{R}}$, as well as any term by term partial derivative with respect to $r$ and $\theta$. Moreover $u^{\mathrm{pr}}$ and $u^{\mathrm{ev}}$ are related to $u$ by the formulas

$$
u^{\mathrm{pr}}=\frac{1}{2}\left(u-\frac{\Delta u}{k^{2}}\right) \quad \text { and } \quad u^{\mathrm{ev}}=\frac{1}{2}\left(u+\frac{\Delta u}{k^{2}}\right)
$$

and satisfy respectively

$$
\Delta u^{\mathrm{pr}}+k^{2} u^{\mathrm{pr}}=0 \quad \text { and } \quad \Delta u^{\mathrm{ev}}-k^{2} u^{\mathrm{ev}}=0 \quad \text { in } \mathbb{R}^{2} \backslash \overline{B_{R}} .
$$

Proof. The starting point is to use the factorization

$$
\Delta^{2} u-k^{4} u=\left(\Delta-k^{2}\right)\left(\Delta+k^{2}\right) u=\left(\Delta+k^{2}\right)\left(\Delta-k^{2}\right) u .
$$

Hence, by defining $u^{\mathrm{pr}}$ and $u^{\mathrm{ev}}$ by (8), we see that the equation $\Delta^{2} u-k^{4} u=0$ is equivalent to the decomposition $u=u^{\mathrm{pr}}+u^{\mathrm{ev}}$ where $u^{\mathrm{pr}}$ and $u^{\mathrm{ev}}$ satisfy (9).

In order to obtain the series expansions (7) of $u^{\mathrm{pr}}$ and $u^{\mathrm{ev}}$, we consider their respective Fourier series with respect to $\theta$. First recall that standard elliptic regularity results tell us that $u^{\mathrm{pr}}$ and $u^{\mathrm{ev}}$ are infinitely differentiable in $\mathbb{R}^{2} \backslash \overline{B_{R}}$. We then easily deduce from (9) that their Fourier coefficients are solutions to Bessel type equations with respect to $r$, which yields the existence of four families of complex numbers $\alpha_{m}^{(1)}, \alpha_{m}^{(2)}, \beta_{m}^{(1)}$ and $\beta_{m}^{(2)}$, for $m \in \mathbb{Z}$, such that

$$
\begin{aligned}
& u^{\mathrm{pr}}(r, \theta)=\sum_{m \in \mathbb{Z}} u_{m}^{\mathrm{pr}}(r) \xi_{m}(\theta) \quad \text { where } \quad u_{m}^{\mathrm{pr}}(r):=\alpha_{m}^{(1)} H_{m}^{(1)}(k r)+\alpha_{m}^{(2)} H_{m}^{(2)}(k r), \\
& u^{\mathrm{ev}}(r, \theta)=\sum_{m \in \mathbb{Z}} u_{m}^{\mathrm{ev}}(r) \xi_{m}(\theta) \quad \text { where } \quad u_{m}^{\mathrm{ev}}(r):=\beta_{m}^{(1)} H_{m}^{(1)}(\mathrm{i} k r)+\alpha_{m}^{(2)} H_{m}^{(2)}(\mathrm{i} k r),
\end{aligned}
$$


where $H_{m}^{(1)}$ and $H_{m}^{(2)}$ are the Hankel functions of order $m$ of the first and second kinds, respectively. As both functions $u^{\mathrm{pr}}$ and $u^{\mathrm{ev}}$ belong to $C^{\infty}\left(\mathbb{R}^{2} \backslash \overline{B_{R}}\right)$, these series are absolutely and uniformly convergent on compact subsets of $\mathbb{R}^{2} \backslash \overline{B_{R}}$, as well as any term by term partial derivative with respect to $r$ and $\theta$. As a consequence, by Parseval's equality, we see that for all $r>R$,

$$
\begin{aligned}
\int_{\partial B_{r}}\left|\frac{\partial u}{\partial n}-\mathrm{i} k u\right|^{2} \mathrm{~d} s & =\sum_{m \in \mathbb{Z}} h_{m}(r), \quad \text { where } \\
h_{m}(r) & :=r\left|\left(\frac{\mathrm{d}}{\mathrm{d} r}-\mathrm{i} k\right)\left(u_{m}^{\mathrm{pr}}+u_{m}^{\mathrm{ev}}\right)\right|^{2} .
\end{aligned}
$$

Using the following asymptotic behavior of Hankel functions [15, Chap. 7, §4.1] which is valid for $|z| \rightarrow \infty$ with $\arg z \in[-\pi / 2,+\pi / 2]$ and fixed order $m \in \mathbb{N}$ :

$$
H_{m}^{(j)}(z)=\sqrt{\frac{2}{\pi z}} \mathrm{e}^{(-1)^{j-1} \mathrm{i}(z-m \pi / 2-\pi / 4)}\left(1+\mathscr{O}\left(z^{-1}\right)\right) \quad \text { for } j=1,2,
$$

as well as the recurrence relation $[1, \S 9.1 .27]$

$$
\left(H_{m}^{(j)}\right)^{\prime}(z)=H_{m-1}^{(j)}(z)-\frac{m}{z} H_{m}^{(j)}(z)
$$

we infer that, as $r \rightarrow+\infty$,

$$
\begin{aligned}
& \left|\left(\frac{\mathrm{d}}{\mathrm{d} r}-\mathrm{i} k\right) H_{m}^{(1)}(k r)\right|=\mathscr{O}\left(r^{-3 / 2}\right) \quad \text { and } \quad\left|\left(\frac{\mathrm{d}}{\mathrm{d} r}-\mathrm{i} k\right) H_{m}^{(2)}(k r)\right| \sim \sqrt{\frac{8 k}{\pi r}} \\
& \left|\left(\frac{\mathrm{d}}{\mathrm{d} r}-\mathrm{i} k\right) H_{m}^{(1)}(\mathrm{i} k r)\right|=\mathscr{O}\left(\mathrm{e}^{-k r}\right) \text { and } \quad\left|\left(\frac{\mathrm{d}}{\mathrm{d} r}-\mathrm{i} k\right) H_{m}^{(2)}(\mathrm{i} k r)\right| \sim \sqrt{\frac{8 k}{\pi r}} \mathrm{e}^{k r} .
\end{aligned}
$$

As a consequence, if there exists $m \in \mathbb{Z}$ such that $\beta_{m}^{(2)} \neq 0$, we will have $h_{m}(r) \gtrsim \mathrm{e}^{2 k r}$ and $u$ will not satisfy the radiation condition, so $\beta_{m}^{(2)}=0$ for all $m \in \mathbb{Z}$. In the same way, if $\alpha_{m}^{(2)} \neq 0$ for some $m \in \mathbb{Z}$, we will have $h_{m}(r) \gtrsim 1$ and $u$ will not satisfy the radiation condition, so $\alpha_{m}^{(2)}=0$ for all $m \in \mathbb{Z}$.

Proposition 4. Let $\alpha_{m}$ and $\beta_{m}$ for $m \in \mathbb{Z}$ be two families of complex numbers such that

$$
\sup _{m \in \mathbb{Z}}\left|\alpha_{m}\right|\left|H_{m}^{(1)}(k R)\right|<\infty \quad \text { and } \sup _{m \in \mathbb{Z}}\left|\beta_{m}\right|\left|H_{m}^{(1)}(\mathrm{i} k R)\right|<\infty
$$

for some $R>0$. Then both series in (7) are absolutely and uniformly convergent on compact subsets of $\mathbb{R}^{2} \backslash \overline{B_{R}}$, as well as any term by term partial derivative with respect to $r$ and $\theta$. Moreover, their respective asymptotic behaviors as $r \rightarrow+\infty$ are

$$
\left|u^{\mathrm{pr}}(r, \theta)\right|=\mathscr{O}\left(r^{-1 / 2}\right) \quad \text { and } \quad\left|u^{\mathrm{ev}}(r, \theta)\right|=\mathscr{O}\left(\mathrm{e}^{-k r}\right),
$$

uniformly with respect to $\theta \in[0,2 \pi)$, and the same holds true for any partial derivative with respect to $r$ and $\theta$. Finally, the function $u:=u^{\mathrm{pr}}+u^{\mathrm{ev}}$ is a radiating solution to $\Delta^{2} u-k^{4} u=0$ in $\mathbb{R}^{2} \backslash \overline{B_{R}}$.

Proof. The absolute and uniform convergence of both series in (7) is a straightforward consequence of the asymptotic behavior of Hankel functions for large order, which is recalled below for positive order $m$ (the case of negative order follows from the relation $\left.H_{-m}^{(1)}(z)=(-1)^{m} H_{m}^{(1)}(z)\right)$. From the series representations of Bessel functions (see $[1, \S 9.1 .10 \& 9.1 .11]$ ), we deduce that

$$
H_{m}^{(1)}(z)=\frac{(m-1) !}{\mathrm{i} \pi}\left(\frac{2}{z}\right)^{m}\left(1+\mathscr{O}\left(m^{-1}\right)\right) \text { as } m \rightarrow+\infty,
$$

uniformly with respect to $z$ in any compact subset of $\mathbb{C} \backslash \mathbb{R}^{-}$. We deduce from this asymptotic behavior that

$$
\frac{H_{m-1}^{(1)}(z)}{H_{m}^{(1)}(z)}=\frac{z}{2 m}+\mathscr{O}\left(m^{-2}\right) \quad \text { as } m \rightarrow \infty .
$$


Using formula (12), we infer that

$$
\frac{\left(H_{m}^{(1)}\right)^{\prime}(z)}{H_{m}^{(1)}(z)}=-\frac{m}{z}+\frac{z}{2 m}+\mathscr{O}\left(m^{-2}\right) \quad \text { as } m \rightarrow \infty .
$$

Iterating this process, we see that for any given $p \geq 1$,

$$
\frac{\left(H_{m}^{(1)}\right)^{(p)}(z)}{H_{m}^{(1)}(z)}=\left(-\frac{m}{z}\right)^{p}+\mathscr{O}\left(m^{p-1}\right) \quad \text { as } m \rightarrow \infty .
$$

All these asymptotic behaviors are valid uniformly in compact subsets of $\mathbb{C} \backslash \mathbb{R}^{-}$.

We now come back to the convergence of the series in (7). For $u^{\mathrm{pr}}$, simply notice that for $M \in \mathbb{N}$,

$$
\sum_{m=-M}^{M}\left|\alpha_{m} H_{m}^{(1)}(k r) \xi_{m}(\theta)\right| \lesssim\left(\sup _{|m| \leq M}\left|\alpha_{m}\right|\left|H_{m}^{(1)}(k R)\right|\right) \sum_{m=-M}^{M}\left|\frac{H_{m}^{(1)}(k r)}{H_{m}^{(1)}(k R)}\right| .
$$

Thanks to assumption (13), the first term of the right-hand side remains bounded as $M \rightarrow+\infty$, and so is the second one, since the asymptotic behavior (15) shows that

$$
\sum_{m=-M}^{M}\left|\frac{H_{m}^{(1)}(k r)}{H_{m}^{(1)}(k R)}\right| \lesssim \sum_{m=-M}^{M}\left(\frac{R}{r}\right)^{|m|} .
$$

The same arguments are still valid for $u^{\text {ev }}$ (replacing $k$ by i $k$ ), as well as for any term by term partial derivative of these series. Indeed, if we derive $p$ times with respect to $r$ and $q$ times with respect to $\theta$, the above inequality has to be replaced by

$$
\sum_{m=-M}^{M}\left|m^{q} \frac{\left(H_{m}^{(1)}\right)^{(p)}(k r)}{H_{m}^{(1)}(k R)}\right| \lesssim \sum_{m=-M}^{M}|m|^{p+q}\left(\frac{R}{r}\right)^{|m|},
$$

using the fact that $\xi_{m}^{\prime}=\mathrm{i} m \xi_{m}$ and the asymptotic behavior (17). As a consequence, both functions $u^{\mathrm{pr}}$ and $u^{\mathrm{ev}}$ belong to $C^{\infty}\left(\mathbb{R}^{2} \backslash \overline{B_{R}}\right)$. Applying respectively the operators $\Delta+k^{2}$ and $\Delta-k^{2}$ to both series in (7) yields (9), which shows by (10) that $u:=u^{\mathrm{pr}}+u^{\mathrm{ev}}$ satisfies $\Delta^{2} u-k^{4} u=0$ in $\mathbb{R}^{2} \backslash \overline{B_{R}}$.

On the other hand, the asymptotic behaviors of $u^{\mathrm{pr}}$ and $u^{\mathrm{ev}}$ for large $r$, in particular the fact these series satisfy the radiation condition, cannot be deduced easily from a direct study of the series. This can be done by a cumbersome analysis of the series, as shown in [10] for a similar water wave scattering problem. However, there is an alternative (and more elegant!) approach which consists in rewriting these series as integral representations of $u^{\mathrm{pr}}$ and $u^{\mathrm{ev}}$, since the latter are well suited to extract their asymptotic behaviors for large $r$. This approach is extensively described in [7, Chap. 2] for the three-dimensional Helmholtz equation and briefly explained in $[7, \S 3.4]$ for the two-dimensional case. We recall here the basic steps for $u^{\mathrm{pr}}$.

Starting from the series representation (7) of $u^{\mathrm{pr}}$, the idea is to show that for any fixed $R^{\prime}>R$,

$$
u^{\mathrm{pr}}(x)=\int_{\partial B_{R^{\prime}}}\left\{u^{\mathrm{pr}}(y) \frac{\partial G^{\mathrm{pr}}}{\partial n_{y}}(x, y)-\frac{\partial u^{\mathrm{pr}}}{\partial n}(y) G^{\mathrm{pr}}(x, y)\right\} \mathrm{d} s_{y} \quad \text { for }|x|>|y|,
$$

where $G^{\mathrm{pr}}$ is the radiating Green's function of the Helmholtz equation, given by

$$
G^{\mathrm{pr}}(x, y)=\frac{\mathrm{i}}{4} H_{0}^{(1)}(k|x-y|),
$$

and $n$ denotes the unit normal vector to $\partial B_{R^{\prime}}$ directed into the exterior of $B_{R^{\prime}}$. The key tool is the famous Graf's addition theorem $[1, \S 9.1 .79]$

$$
G^{\mathrm{pr}}(x, y)=\frac{\mathrm{i} \pi}{2} \sum_{m \in \mathbb{Z}} H_{m}^{(1)}(k|x|) \xi_{m}\left(\theta_{x}\right) J_{m}(k|y|) \overline{\xi_{m}\left(\theta_{y}\right)} \quad \text { for }|x|>|y|,
$$


where $J_{m}$ is the Bessel function of order $m$ [1] and $\theta_{x}$ (respectively, $\theta_{y}$ ) denotes the polar angle of $x$ (respectively, $y$ ). As above, this series is absolutely and uniformly convergent on compact subsets of $|x|>|y|$, as well as any term by term partial derivative with respect to $x$ or $y$. If we insert this formula as well as the series (7) for all $y \in \partial B_{R^{\prime}}$ into the integral of the right-hand side of (18) and use the orthonormality of the $\xi_{m}$ in $L^{2}\left(S^{1}\right)$, this integral can be written as

$$
\frac{\mathrm{i} \pi k R^{\prime}}{2} \sum_{m \in \mathbb{Z}} \alpha_{m}\left\{H_{m}^{(1)}\left(k R^{\prime}\right) J_{m}^{\prime}\left(k R^{\prime}\right)-\left(H_{m}^{(1)}\right)^{\prime}\left(k R^{\prime}\right) J_{m}\left(k R^{\prime}\right)\right\} H_{m}^{(1)}(k|x|) \xi_{m}\left(\theta_{x}\right) .
$$

The term between braces is the wronskian of $H_{m}^{(1)}$ and $J_{m}$. It is explicitly given by $[1, \S 9.1 .16]$

$$
H_{m}^{(1)}\left(k R^{\prime}\right) J_{m}^{\prime}\left(k R^{\prime}\right)-\left(H_{m}^{(1)}\right)^{\prime}\left(k R^{\prime}\right) J_{m}\left(k R^{\prime}\right)=\frac{2}{\mathrm{i} \pi k R^{\prime}},
$$

which shows that the integral of the right-hand side of (18) is exactly the series (7) of $u^{\mathrm{pr}}(x)$. Hence (18) is proved. Replacing $k$ by $\mathrm{i} k$, we obtain in the same way the integral representation of $u^{\mathrm{ev}}$, which involves the Green's function

$$
G^{\mathrm{ev}}(x, y)=\frac{\mathrm{i}}{4} H_{0}^{(1)}(\mathrm{i} k|x-y|) .
$$

Using the asymptotic behavior (11), we infer that

$$
\left|G^{\mathrm{pr}}(x, y)\right|=\mathscr{O}\left(|x|^{-1 / 2}\right) \quad \text { and } \quad\left|G^{\mathrm{ev}}(x, y)\right|=\mathscr{O}\left(\mathrm{e}^{-k|x|}\right) \quad \text { as }|x| \rightarrow+\infty,
$$

uniformly with respect to $\theta_{x} \in[0,2 \pi)$ and $y \in \partial B_{R^{\prime}}$. Using (12), we see that the same holds true for any partial derivative of these Green's functions with respect to $x$ or $y$. These estimates together with the integral representation of $u^{\mathrm{pr}}$ and $u^{\mathrm{ev}}$ yield (14). Finally, the same arguments show that

$$
\left|\frac{\partial}{\partial|x|} G^{\mathrm{pr}}(x, y)-\mathrm{i} k G^{\mathrm{pr}}(x, y)\right|=\mathscr{O}\left(|x|^{-3 / 2}\right),
$$

uniformly with respect to $\theta_{x} \in[0,2 \pi)$ and $y \in \partial B_{R^{\prime}}$, from which we deduce that $u^{\text {pr }}$ satisfies the radiation condition (6). This obviously holds true for $u^{\mathrm{ev}}$, since it is exponentially decreasing, as well as its derivatives. Hence $u:=u^{\mathrm{pr}}+u^{\mathrm{ev}}$ is a radiating solution to $\Delta^{2} u-k^{4} u=0$. This completes the proof.

\section{Uniqueness}

We now address the question of uniqueness in problem (1): if $u^{\mathrm{i}}=0$, does a solution $v^{\mathrm{s}} \in H_{\mathrm{loc}}^{2}(\Omega)$ to problem (1) vanish in $\Omega$ ? Throughout this section, we simply denote $v^{\mathrm{s}}=u$. To begin with, we prove that the "propagating part" of $u$ actually vanishes by a standard argument based on the energy flux. In order to introduce such energy flux, we need the following Green formula (see for example [11]).

Lemma 5. In a bounded domain $\mathcal{O}$ of class $C^{2}$, denoting $H^{2}\left(\mathcal{O}, \Delta^{2}\right):=\left\{u \in H^{2}(\mathcal{O}), \Delta^{2} u \in L^{2}(\mathcal{O})\right\}$, the linear mapping $T: u \mapsto(N u, M u)$ is continuous from $H^{2}\left(\mathcal{O}, \Delta^{2}\right)$ to $H^{-3 / 2}(\partial \mathcal{O}) \times H^{-1 / 2}(\partial \mathcal{O})$. Moreover, for all $u \in H^{2}\left(\mathcal{O}, \Delta^{2}\right)$ and $v \in H^{2}(\mathcal{O})$, we have the Green formula

$$
\begin{aligned}
& \int_{\mathcal{O}} \Delta^{2} u \bar{v} \mathrm{~d} x=a(u, v)-\int_{\partial \mathcal{O}}\left(N u \bar{v}+M u \frac{\partial \bar{v}}{\partial n}\right) \mathrm{d} s \text { where } \\
& a(u, v):=\int_{\mathcal{O}}\left\{\nu \Delta u \Delta \bar{v}+(1-\nu) \sum_{i, j=1}^{2} \frac{\partial^{2} u}{\partial x_{i} \partial x_{j}} \frac{\partial^{2} \bar{v}}{\partial x_{i} \partial x_{j}}\right\} \mathrm{d} x .
\end{aligned}
$$

Here, the unit normal vector to $\partial \mathcal{O}$, which is involved in the definition (3) of the surface operators $M$ and $N$, is directed into the exterior of $\mathcal{O}$. The first part of the integral on $\partial \mathcal{O}$ has the meaning of duality pairing between $H^{3 / 2}(\partial \mathcal{O})$ and $H^{-3 / 2}(\partial \mathcal{O})$ while the second part has the meaning of duality pairing between $H^{1 / 2}(\partial \mathcal{O})$ and $H^{-1 / 2}(\partial \mathcal{O})$. 
Let us consider the annulus $B_{r, r^{\prime}}:=B_{r^{\prime}} \backslash \overline{B_{r}}$ with $r^{\prime}>r$ for sufficiently large $r$ (so that $B_{r, r^{\prime}} \subset \Omega$ ). Starting from

$$
\int_{B_{r, r^{\prime}}}\left(\Delta^{4} u-k^{4} u\right) \bar{u} \mathrm{~d} x=0
$$

and applying the Green formula of Lemma 5 in the domain $B_{r, r^{\prime}}$ for $v=u$, we obtain that the quantity

$$
\mathcal{F}(u):=\operatorname{Im}\left\{\int_{\partial B_{r}}\left(N u \bar{u}+M u \frac{\partial \bar{u}}{\partial n}\right) \mathrm{d} s\right\},
$$

where the unit normal vector to $\partial B_{r}$ is directed into the exterior of $B_{r}$, does not depend on $r$. It is called the energy flux.

We are now in a position to prove that the propagating part $u^{\mathrm{pr}}$ of $u$ vanishes.

Proposition 6. Let us consider a solution u to problem (1) corresponding to $u^{\mathrm{i}}=0$. Then $u^{\mathrm{pr}}=0$ where $u^{\mathrm{pr}}$ is defined by (8).

Proof. Let us introduce the bounded domain $\Omega_{r}=\Omega \cap B_{r}$, where $r>0$ is sufficiently large to contain the obstacle $\mathcal{D}$, and write

$$
\int_{\Omega_{r}}\left(\Delta^{2} u-k^{4} u\right) \bar{u} \mathrm{~d} x=0 .
$$

Next we use Lemma 5 for $\mathcal{O}=\Omega_{r}$, which implies that

$$
a(u, u)-k^{4}\|u\|_{L^{2}\left(\Omega_{r}\right)}^{2}-\int_{\partial B_{r}}\left(N u \bar{u}+M u \frac{\partial \bar{u}}{\partial n}\right) \mathrm{d} s=0,
$$

for any of the four boundary conditions that we consider on $\partial \Omega$. We conclude that for sufficiently large $r$,

$$
\operatorname{Im}\left\{\int_{\partial B_{r}}\left(N u \bar{u}+M u \frac{\partial \bar{u}}{\partial n}\right) \mathrm{d} s\right\}=0,
$$

that is the energy flux $\mathcal{F}(u)$ vanishes. By using the decomposition $u=u^{\mathrm{pr}}+u^{\mathrm{ev}}$ and the asymptotic behavior when $r \rightarrow+\infty$ of $u^{\mathrm{pr}}$ and $u^{\mathrm{ev}}$ given by (14), we infer that

$$
\mathcal{F}(u)=\lim _{r \rightarrow+\infty} \mathcal{F}\left(u^{\mathrm{pr}}\right) .
$$

To obtain an asymptotic behavior of $\mathcal{F}\left(u^{\mathrm{pr}}\right)$ when $r \rightarrow+\infty$, let us compute $N u^{\mathrm{pr}}$ and $M u^{\mathrm{pr}}$ on $\partial B_{r}$. We first rewrite the definition (3) of $M$ and $N$ in polar coordinates as follows

$$
\left\{\begin{aligned}
M u & =\Delta u-(1-\nu)\left(\frac{1}{r} \frac{\partial u}{\partial r}+\frac{1}{r^{2}} \frac{\partial^{2} u}{\partial \theta^{2}}\right) \\
N u & =-\frac{\partial}{\partial r} \Delta u-(1-\nu) \frac{1}{r^{2}} \frac{\partial}{\partial \theta}\left(\frac{\partial^{2} u}{\partial r \partial \theta}-\frac{1}{r} \frac{\partial u}{\partial \theta}\right) .
\end{aligned}\right.
$$

That $\Delta u^{\mathrm{pr}}+k^{2} u^{\mathrm{pr}}=0$ in $\Omega$ and making use once again of (14) imply that

$$
M u^{\mathrm{pr}}=-k^{2} u^{\mathrm{pr}}+\mathscr{O}\left(r^{-3 / 2}\right) \quad \text { and } \quad N u^{\mathrm{pr}}=k^{2} \frac{\partial u^{\mathrm{pr}}}{\partial r}+\mathscr{O}\left(r^{-5 / 2}\right) .
$$

We obtain that

$$
\begin{aligned}
\mathcal{F}\left(u^{\mathrm{pr}}\right) & =k^{2} \operatorname{Im}\left\{\int_{\partial B_{r}}\left(\frac{\partial u^{\mathrm{pr}}}{\partial r} \overline{u^{\mathrm{pr}}}-u^{\mathrm{pr}} \frac{\partial \overline{u^{\mathrm{pr}}}}{\partial r}\right) \mathrm{d} s\right\}+\mathscr{O}\left(r^{-1}\right) \\
& =2 k^{2} \operatorname{Im}\left\{\int_{\partial B_{r}} \frac{\partial u^{\mathrm{pr}}}{\partial r} \overline{u^{\mathrm{pr}}} \mathrm{d} s\right\}+\mathscr{O}\left(r^{-1}\right) \\
& =2 k^{3} \int_{\partial B_{r}}\left|u^{\mathrm{pr}}\right|^{2} \mathrm{~d} s+2 k^{2} \operatorname{Im}\left\{\int_{\partial B_{r}}\left(\frac{\partial u^{\mathrm{pr}}}{\partial r}-\mathrm{i} k u^{\mathrm{pr}}\right) \overline{u^{\mathrm{pr}}} \mathrm{d} s\right\}+\mathscr{O}\left(r^{-1}\right) .
\end{aligned}
$$


From (14) and the radiation condition, we have

$$
\int_{\partial B_{r}}\left|u^{\mathrm{pr}}\right|^{2} \mathrm{~d} s=\mathscr{O}(1) \quad \text { and } \quad \lim _{r \rightarrow+\infty} \int_{\partial B_{r}}\left|\frac{\partial u^{\mathrm{pr}}}{\partial r}-\mathrm{i} k u^{\mathrm{pr}}\right|^{2} \mathrm{~d} s=0,
$$

hence we end up with

$$
\mathcal{F}(u)=2 k^{3} \lim _{r \rightarrow+\infty} \int_{\partial B_{r}}\left|u^{\mathrm{pr}}\right|^{2} \mathrm{~d} s=0 .
$$

The so-called Rellich Lemma (see for example [6, Theorem 3.5]) tells us that the only possible solution $u^{\mathrm{pr}} \in H_{\mathrm{loc}}^{1}(\Omega)$ to the Helmholtz equation $\Delta u^{\mathrm{pr}}+k^{2} u^{\mathrm{pr}}=0$ in $\Omega$ which satisfies the latter asymptotic behavior at infinity is $u^{\mathrm{pr}}=0$.

Proposition 6 is independent of the boundary conditions on $\partial \Omega$. Uniqueness in three cases of boundary conditions out of four can then be easily obtained.

Theorem 7. For $\left(\mathcal{B}_{1}, \mathcal{B}_{2}\right)=(I, \partial / \partial n)$ (clampled plate), $\left(\mathcal{B}_{1}, \mathcal{B}_{2}\right)=(I, M)$ (simply supported plate) or $\left(\mathcal{B}_{1}, \mathcal{B}_{2}\right)=(\partial / \partial n, N)$ (roller supported plate), problem (1) has at most one solution.

Proof. Let us consider a solution $u$ to problem (1) for $u^{\mathrm{i}}=0$. From Proposition 6 we have $u=u^{\mathrm{ev}}$, in other words $\Delta u-k^{2} u=0$ in $\Omega$. By multiplying this equation by $\bar{u}$, integrating over $\Omega_{r}$ and using Green's formula, we obtain

$$
-\int_{\Omega_{r}}\left(|\nabla u|^{2}+k^{2}|u|^{2}\right) \mathrm{d} x+\int_{\partial B_{r}} \frac{\partial u}{\partial n} \bar{u} \mathrm{~d} s+\int_{\partial \Omega} \frac{\partial u}{\partial n} \bar{u} \mathrm{~d} s=0 .
$$

Given that in the three cases of boundary conditions we have either $\left.u\right|_{\partial \Omega}=0$ or $\left.(\partial u / \partial n)\right|_{\partial \Omega}=0$, the integral on $\partial \Omega$ vanishes. In addition, using the Cauchy-Schwarz inequality and the asymptotic behavior of $u^{\mathrm{ev}}$ given by (14), we have

$$
\lim _{r \rightarrow+\infty} \int_{\partial B_{r}} \frac{\partial u}{\partial n} \bar{u} \mathrm{~d} s=0 .
$$

Passing to the limit $r \rightarrow+\infty$ in the identity (22), we get

$$
\int_{\Omega}\left(|\nabla u|^{2}+k^{2}|u|^{2}\right) \mathrm{d} x=0,
$$

so that $u=0$ in $\Omega$, which completes the proof.

The fourth case of boundary condition $\left(\mathcal{B}_{1}, \mathcal{B}_{2}\right)=(M, N)$ (free plate) is more complicated. We begin with a general result which is weaker than Theorem 7 , since we have to exclude a countable set of wave numbers $k$ which accumulates at $+\infty$.

Theorem 8. The problem (1) for $\left(\mathcal{B}_{1}, \mathcal{B}_{2}\right)=(M, N)$ (free plate) has at most one solution for all $k>0$, except for at most a countable set of numbers $k_{n}>0, n \in \mathbb{N}$, such that $k_{n} \rightarrow+\infty$.

Proof of Theorem 8. Let us consider a solution $u$ to problem (1) for $u^{\mathrm{i}}=0$. From Proposition 6 we have $\Delta u-k^{2} u=0$ in $\Omega$. That $u \in H^{2}\left(\Omega_{R}\right)$ for sufficiently large $R$ implies that $\left.u\right|_{\partial \Omega} \in H^{1}(\partial \Omega)$. Since $\Omega$ is of class $C^{3}$, by standard regularity results for elliptic equations [5] we additionally have that $u \in H^{3}\left(\Omega_{R}\right)$, hence $\left.(\partial u / \partial n)\right|_{\partial \Omega} \in H^{1}(\partial \Omega)$. As a consequence of $\Delta u-k^{2} u=0$ in $\Omega, M u=0$ and $N u=0$ on $\partial \Omega$ as well as formulas (3), the functions $U:=\left.u\right|_{\partial \Omega}$ and $V:=\left.(\partial u / \partial n)\right|_{\partial \Omega}$ are solutions to the following problem on $\partial \Omega$ : find $(U, V) \in H^{1}(\partial \Omega)^{2}$ such that

$$
\left\{\begin{array}{l}
\frac{\mathrm{d}^{2} U}{\mathrm{~d} s^{2}}+\gamma V=\lambda U, \\
-\frac{\mathrm{d}^{2} V}{\mathrm{~d} s^{2}}+\frac{\mathrm{d}}{\mathrm{d} s}\left(\gamma \frac{\mathrm{d} U}{\mathrm{~d} s}\right)=\lambda V,
\end{array}\right.
$$


where we have introduced $\lambda:=k^{2} /(1-\nu)$. Such coupled system can be seen as a non-selfadjoint eigenvalue problem. Let us rewrite the system (23) as

$$
\left\{\begin{array}{l}
\left(-\frac{\mathrm{d}^{2} U}{\mathrm{~d} s^{2}}+U\right)-(1-\lambda) U-\gamma V=0, \\
\left(-\frac{\mathrm{d}^{2} V}{\mathrm{~d} s^{2}}+V\right)+\gamma^{\prime}(s) \frac{\mathrm{d} U}{\mathrm{~d} s}+\lambda \gamma U-\left(1+\gamma^{2}+\lambda\right) V=0,
\end{array}\right.
$$

which itself can be rewritten in the form of a weak formulation: find $(U, V) \in H^{1}(\partial \Omega)^{2}$ such that for all $(\tilde{U}, \tilde{V}) \in H^{1}(\partial \Omega)^{2}$,

$$
\left(\left(\begin{array}{c}
U \\
V
\end{array}\right),\left(\begin{array}{c}
\tilde{U} \\
\tilde{V}
\end{array}\right)\right)_{H^{1}(\partial \Omega)^{2}}-\left(\mathcal{J}(\lambda)\left(\begin{array}{c}
U \\
V
\end{array}\right),\left(\begin{array}{c}
\tilde{U} \\
\tilde{V}
\end{array}\right)\right)_{L^{2}(\partial \Omega)^{2}}=0
$$

where the operator $\mathcal{J}(\lambda)$ on $H^{1}(\partial \Omega)^{2}$ is defined as

$$
\mathcal{J}(\lambda):=\left(\begin{array}{cc}
1-\lambda & \gamma \\
-\gamma^{\prime}(s) \frac{\mathrm{d}}{\mathrm{d} s}-\lambda \gamma & 1+\gamma^{2}+\lambda
\end{array}\right) .
$$

Introducing $\mathcal{X}=(U, V), \tilde{\mathcal{X}}=(\tilde{U}, \tilde{V})$ and the operator $\mathcal{K}(\lambda)$ such that for all $\mathcal{X}$ and $\tilde{\mathcal{X}}$ in $H^{1}(\partial \Omega)^{2}$, $(\mathcal{K}(\lambda) \mathcal{X}, \tilde{\mathcal{X}})_{H^{1}(\partial \Omega)^{2}}=(\mathcal{J}(\lambda) \mathcal{X}, \mathcal{X})_{L^{2}(\partial \Omega)^{2}}$, such weak formulation also reads as the equation $\mathcal{X}-\mathcal{K}(\lambda) \mathcal{X}=$ 0 in $H^{1}(\partial \Omega)^{2}$, where $\lambda \mapsto \mathcal{K}(\lambda)$ is obviously a family of compact operators on $H^{1}(\partial \Omega)^{2}$ which analytically depends on $\lambda \in \mathbb{C}$. From the Analytic Fredholm theorem (see [7, Theorem 8.26] or [18]), we can conclude that $\mathcal{X}=0$ except for a countable set of $\mathbb{C}$ if we can find a particular point $\lambda \in \mathbb{C}$ such that the operator $I-\mathcal{K}(\lambda)$ is injective. Then we will obtain that except for a countable set of positive $k_{n} \in \mathbb{R}$ such that $k_{n} \rightarrow+\infty$ when $n \rightarrow+\infty$, we have $\left(\left.u\right|_{\partial \Omega},\left.(\partial u / \partial n)\right|_{\partial \Omega}\right)=(0,0)$, which implies that $u=0$ in $\Omega$.

Let us find some $\mu \in \mathbb{R}$ such that $I-\mathcal{K}(\mathrm{i} \mu)$ is injective. In the remainder of the proof, we will use the short notation $U^{\prime}=\mathrm{d} U / \mathrm{d} s$, while $(\cdot, \cdot)$ and $\|\cdot\|$ will denote the scalar product and the norm of $L^{2}(\partial \Omega)$, respectively. Multiplying the system $(23)$ by $(U, V)^{T}$ in the sense of the scalar product $(\cdot, \cdot)$ and integrating by parts, we get

$$
\left\{\begin{array}{l}
\left\|U^{\prime}\right\|^{2}+\mathrm{i} \mu\|U\|^{2}=(\gamma V, U) \\
\left\|V^{\prime}\right\|^{2}-\mathrm{i} \mu\|V\|^{2}=\left(\gamma U^{\prime}, V^{\prime}\right)
\end{array}\right.
$$

which implies that

$$
\left\{\begin{array}{l}
\left\|U^{\prime}\right\|^{4}+\mu^{2}\|U\|^{4}=|(\gamma V, U)|^{2} \lesssim\|U\|^{2}\|V\|^{2} \\
\left\|V^{\prime}\right\|^{4}+\mu^{2}\|V\|^{4}=\left|\left(\gamma U^{\prime}, V^{\prime}\right)\right|^{2} \lesssim\left\|U^{\prime}\right\|^{2}\left\|V^{\prime}\right\|^{2}
\end{array}\right.
$$

From the first inequality of (24), we obtain that there exists $C>0$ such that

$$
\left\|U^{\prime}\right\|^{4}+\mu^{2}\|U\|^{4} \leq C\left(\|U\|^{4}+\|V\|^{4}\right),
$$

which induces that if $\mu^{2} \geq C$, we get $\left\|U^{\prime}\right\|^{4} \leq C\|V\|^{4}$. Plugging such estimate in the second inequality of (24) yields

$$
\left\|V^{\prime}\right\|^{4}+\mu^{2}\|V\|^{4} \lesssim\|V\|^{2}\left\|V^{\prime}\right\|^{2} \lesssim|\mu|\|V\|^{4}+|\mu|^{-1}\left\|V^{\prime}\right\|^{4} .
$$

Taking $|\mu|$ sufficiently large, we finally obtain $V=0$, and then $U=0$, which completes the proof.

We complement Theorem 8 with a result for sufficiently large values of $k$ when the obstacle $\mathcal{D}$ is convex.

Theorem 9. Let $L$ denote the perimeter of D. Assume that

$$
\begin{aligned}
& \text { there exists } \gamma_{0}<0 \text { such that } \gamma(s) \leq \gamma_{0} \text { for all } s \in[0, L] \text { and } \\
& \sup _{s \in[0, L]}\left|\frac{\gamma^{\prime}(s)}{\gamma(s)}\right| \leq \frac{2 k}{\sqrt{1-\nu}} .
\end{aligned}
$$

Then problem $(1)$ for $\left(\mathcal{B}_{1}, \mathcal{B}_{2}\right)=(M, N)$ (free plate) has at most one solution. 
Remark 10. The first assumption of Theorem 9, that is (25), implies in particular that $\gamma(s) \leq 0$ for all $s \in[0, L]$, which means that the obstacle $\mathcal{D}$ is convex. The assumption (26) means that the obstacle has a slowly varying curvature or that $k$ is large. In particular, if $\mathcal{D}$ is a disk, then uniqueness holds for any $k>0$.

Proof of Theorem 9. Considering again a solution $u$ to problem (1) for $u^{\mathrm{i}}=0$ and proceeding as in the proof of Theorem 7 , we obtain by passing to the limit $r \rightarrow+\infty$ in (22) that

$$
\int_{\Omega}\left(|\nabla u|^{2}+k^{2}|u|^{2}\right) \mathrm{d} x=\int_{\partial \Omega} \frac{\partial u}{\partial n} \bar{u} \mathrm{~d} s .
$$

As a consequence of $M u=0$ on $\partial \Omega$, the first formula in (3) and $\Delta u-k^{2} u=0$ in $\Omega$, we have on $\partial \Omega$ :

$$
k^{2} u-(1-\nu)\left(\frac{\partial^{2} u}{\partial s^{2}}+\gamma \frac{\partial u}{\partial n}\right)=0 .
$$

Hence

$$
\begin{aligned}
\int_{\Omega}\left(|\nabla u|^{2}+k^{2}|u|^{2}\right) \mathrm{d} x & =\int_{\partial \Omega} \frac{1}{\gamma(s)}\left(\frac{k^{2}}{1-\nu} u-\frac{\partial^{2} u}{\partial s^{2}}\right) \bar{u} \mathrm{~d} s \\
& =\int_{\partial \Omega} \frac{1}{\gamma(s)} \frac{k^{2}}{1-\nu}|u|^{2}+\frac{\partial u}{\partial s} \frac{\partial}{\partial s}\left(\frac{1}{\gamma} \bar{u}\right) \mathrm{d} s \\
& =\int_{\partial \Omega} \frac{1}{\gamma(s)}\left(\frac{k^{2}}{1-\nu}|u|^{2}+\left|\frac{\partial u}{\partial s}\right|^{2}-\frac{\gamma^{\prime}(s)}{\gamma(s)} \frac{\partial u}{\partial s} \bar{u}\right) \mathrm{d} s .
\end{aligned}
$$

We have that for all $s \in[0, L]$,

$$
\operatorname{Re}\left\{\frac{k^{2}}{1-\nu}|u|^{2}+\left|\frac{\partial u}{\partial s}\right|^{2}-\frac{\gamma^{\prime}(s)}{\gamma(s)} \frac{\partial u}{\partial s} \bar{u}\right\} \geq \frac{k^{2}}{1-\nu}|u|^{2}+\left|\frac{\partial u}{\partial s}\right|^{2}-\left|\frac{\gamma^{\prime}(s)}{\gamma(s)}\right|\left|\frac{\partial u}{\partial s}\right||u| .
$$

Since the right-hand side is non negative if $\left|\gamma^{\prime}(s) / \gamma(s)\right|^{2}-4 k^{2} /(1-\nu) \leq 0$, the assumption (26) implies $\int_{\Omega}\left(|\nabla u|^{2}+k^{2}|u|^{2}\right) \mathrm{d} x \leq 0$, which yields $u=0$ in $\Omega$.

\section{The Dirichlet problem outside a ball}

We now consider a particular case of problem (1), namely a circular obstacle $B_{R}$ of radius $R>0$ with a pair of Dirichlet boundary conditions, i.e.,

$$
\begin{cases}\Delta^{2} u-k^{4} u=0 & \text { in } \mathbb{R}^{2} \backslash \overline{B_{R}} \\ \left(u, \frac{\partial u}{\partial n}\right)=(f, g) & \text { on } \partial B_{R}, \\ \lim _{r \rightarrow+\infty} \int_{\partial B_{r}}\left|\frac{\partial u}{\partial n}-\mathrm{i} k u\right|^{2} \mathrm{~d} s=0 . & \end{cases}
$$

Unlike our initial problem (1), we choose here the opposite direction for the unit normal on $\partial B_{R}$ : in this section $n$ denotes the unit normal vector to $\partial B_{R}$ directed into the exterior of $B_{R}$. On the other hand, we keep the same direction for the unit normal on $\partial B_{r}$ involved in the radiation condition: it is also directed into the exterior of $B_{r}$.

We already know by Proposition 3 that if there exists a solution $u$ to (27), it can be expressed by the series expansion (7) which involves two complex sequences $\left(\alpha_{m}\right)$ and $\left(\beta_{m}\right)$, for $m \in \mathbb{Z}$. We first rewrite these series in a more convenient form by introducing two families of functions $\left(F_{m}(r)\right)$ and $\left(G_{m}(r)\right)$ whose definition simply follows from linearity. The idea is to relate the Fourier series of $f$ and $g$,

$$
\begin{aligned}
f=\sum_{m \in \mathbb{Z}} f_{m} \xi_{m} \quad \text { and } \quad g=\sum_{m \in \mathbb{Z}} g_{m} \xi_{m} \text { where } \\
f_{m}:=\frac{1}{R}\left(f, \xi_{m}\right)_{L^{2}\left(\partial B_{R}\right)} \quad \text { and } \quad g_{m}:=\frac{1}{R}\left(g, \xi_{m}\right)_{L^{2}\left(\partial B_{R}\right)},
\end{aligned}
$$


with that of $u$. The latter will simply write as

$$
u(r, \theta)=\sum_{m \in \mathbb{Z}}\left(f_{m} F_{m}(r)+g_{m} G_{m}(r)\right) \xi_{m}(\theta),
$$

if we choose $F_{m}$ and $G_{m}$ so that $F_{m}(r) \xi_{m}(\theta)$ and $G_{m}(r) \xi_{m}(\theta)$ are respectively solutions to (27) for the particular pairs $\left(\xi_{m}, 0\right)$ and $\left(0, \xi_{m}\right)$ of Dirichlet data on $\partial B_{R}$. Note that $\xi_{m}$, initially defined in (4) as a unit function of $L^{2}\left(S^{1}\right)$, is trivially identified here with a function of $L^{2}\left(\partial B_{R}\right)$ (that is no longer unit, which explains the factor $R^{-1}$ in the expression (28) of the Fourier coefficients). It follows from (7) that $F_{m}(r)$ and $G_{m}(r)$ are both linear combinations of $H_{m}^{(1)}(k r)$ and $H_{m}^{(1)}(\mathrm{i} k r)$. The coefficients are found by writing the two boundary conditions at $r=R$. We readily obtain

$$
\begin{aligned}
F_{m}(r) & =\frac{1}{r_{m}-s_{m}}\left(-s_{m} \frac{H_{m}^{(1)}(k r)}{H_{m}^{(1)}(k R)}+r_{m} \frac{H_{m}^{(1)}(\mathrm{i} k r)}{H_{m}^{(1)}(\mathrm{i} k R)}\right) \text { and } \\
G_{m}(r) & =\frac{1}{r_{m}-s_{m}}\left(\frac{H_{m}^{(1)}(k r)}{H_{m}^{(1)}(k R)}-\frac{H_{m}^{(1)}(\mathrm{i} k r)}{H_{m}^{(1)}(\mathrm{i} k R)}\right),
\end{aligned}
$$

where we have denoted

$$
r_{m}:=\frac{k\left(H_{m}^{(1)}\right)^{\prime}(k R)}{H_{m}^{(1)}(k R)} \quad \text { and } \quad s_{m}:=\frac{\mathrm{i} k\left(H_{m}^{(1)}\right)^{\prime}(\mathrm{i} k R)}{H_{m}^{(1)}(\mathrm{i} k R)} .
$$

It remains to describe a functional framework in which the series (29) provides us the existence of a solution to our Dirichlet problem (27). This is the subject of the following proposition.

Proposition 11. For all pair $(f, g) \in H^{3 / 2}\left(\partial B_{R}\right) \times H^{1 / 2}\left(\partial B_{R}\right)$, problem (27) has a unique solution $u \in H_{\mathrm{loc}}^{2}\left(\mathbb{R}^{2} \backslash \overline{B_{R}}\right)$ given by the series expansion (29), where the series converges in $H_{\mathrm{loc}}^{2}\left(\mathbb{R}^{2} \backslash \overline{B_{R}}\right)$.

Proof. Most part of the work is already done. First, using the uniqueness Theorem 7 in the clamped case with $\mathcal{D}=B_{R}$, we know that problem (27) has at most one solution. We are going to prove its existence by examining the convergence properties of the series (29). And here again, a non-obvious result is already known: Proposition 4 tells us that this series, as soon as it converges in some sense, defines a radiating solution to $\Delta^{2} u-k^{4} u=0$ in $\mathbb{R}^{2} \backslash \overline{B_{R}}$. The $C^{\infty}$ convergence properties stated in Propositions 3 and 4 are valid outside a vicinity of the boundary $\partial B_{R}$. They follow actually from interior regularity of elliptic equations. Here we need a sharper study of the behavior of the series up to $\partial B_{R}$, that is, exactly where it has the worse convergence.

First recall that thanks to (5), the choice of the trace spaces $H^{3 / 2}\left(\partial B_{R}\right)$ and $H^{1 / 2}\left(\partial B_{R}\right)$ for $f$ and $g$ can be interpreted in terms of convergence of their respective Fourier coefficients (28) as follows:

$$
\sum_{m \in \mathbb{Z}}\left(1+m^{2}\right)^{3 / 2}\left|f_{m}\right|^{2}<+\infty \text { and } \sum_{m \in \mathbb{Z}}\left(1+m^{2}\right)^{1 / 2}\left|g_{m}\right|^{2}<+\infty .
$$

It remains to understand how these assumptions dictate the behavior of the series (29). Recall that convergence in $H_{\text {loc }}^{2}\left(\mathbb{R}^{2} \backslash \overline{B_{R}}\right)$ means convergence in $H^{2}\left(B_{R, R^{\prime}}\right)$ for any annulus $B_{R, R^{\prime}}:=B_{R^{\prime}} \backslash \overline{B_{R}}$ with $R^{\prime}>R$.

We start by proving the convergence in $L^{2}\left(B_{R, R^{\prime}}\right)$ for any $R^{\prime}>R$. Denoting

$$
u_{m}(r, \theta):=\left(f_{m} F_{m}(r)+g_{m} G_{m}(r)\right) \xi_{m}(\theta),
$$

we deduce from the orthogonality of the $\xi_{m}$ in $L^{2}\left(S^{1}\right)$ and Fubini's theorem that the $u_{m}$ are orthogonal in $L^{2}\left(B_{R, R^{\prime}}\right)$. So, by Parseval's identity, for all $m_{0} \in \mathbb{N}$, we have

$$
\begin{aligned}
\left\|\sum_{m=-m_{0}}^{m_{0}} u_{m}\right\|_{L^{2}\left(B_{R, R^{\prime}}\right)}^{2} & =\sum_{m=-m_{0}}^{m_{0}}\left\|u_{m}\right\|_{L^{2}\left(B_{R, R^{\prime}}\right)}^{2}, \quad \text { where } \\
\left\|u_{m}\right\|_{L^{2}\left(B_{R, R^{\prime}}\right)}^{2} & =\int_{R}^{R^{\prime}}\left|f_{m} F_{m}(r)+g_{m} G_{m}(r)\right|^{2} r \mathrm{~d} r .
\end{aligned}
$$


Using formula (15), we infer that

$$
\left|\frac{H_{m}^{(1)}(k r)}{H_{m}^{(1)}(k R)}\right| \lesssim 1 \quad \text { and } \quad\left|\frac{H_{m}^{(1)}(k r)}{H_{m}^{(1)}(k R)}-\frac{H_{m}^{(1)}(\mathrm{i} k r)}{H_{m}^{(1)}(\mathrm{i} k R)}\right| \lesssim \frac{1}{|m|},
$$

for all $m \neq 0$ and $r \in\left(R, R^{\prime}\right)$. Moreover, it follows from (32) and (16) that

$$
r_{m} \sim-\frac{|m|}{R}, \quad s_{m} \sim-\frac{|m|}{R} \quad \text { and } \quad r_{m}-s_{m} \sim \frac{k^{2} R}{|m|} \quad \text { as }|m| \rightarrow+\infty .
$$

Hence by eqs. (30) and (31), where (30) can be rewritten equivalently

$$
F_{m}(r)=\frac{H_{m}^{(1)}(k r)}{H_{m}^{(1)}(k R)}-r_{m} G_{m}(r),
$$

we deduce that $\left|F_{m}(r)\right| \lesssim|m|$ and $\left|G_{m}(r)\right| \lesssim 1$ for $m \neq 0$ and $r \in\left(R, R^{\prime}\right)$, which shows by (35) that

$$
\left\|u_{m}\right\|_{L^{2}\left(B_{R, R^{\prime}}\right)}^{2} \lesssim m^{2}\left|f_{m}\right|^{2}+\left|g_{m}\right|^{2} .
$$

By virtue of the assumptions (33), this yields the convergence of the series (34) as $m_{0} \rightarrow+\infty$. We have thus proved that the series $(29)$ converges in $L^{2}\left(B_{R, R^{\prime}}\right)$.

To see that the convergence holds true in $H^{2}\left(B_{R, R^{\prime}}\right)$, we use Green's formula of Lemma 5 in the domain $B_{R, R^{\prime}}$, which shows that

$$
a\left(u_{m}, u_{m^{\prime}}\right)=k^{4}\left(u_{m}, u_{m^{\prime}}\right)_{L^{2}\left(B_{R, R^{\prime}}\right)}-\int_{\partial B_{R} \cup \partial B_{R^{\prime}}}\left(N u_{m} \overline{u_{m^{\prime}}}+M u_{m} \frac{\partial \overline{u_{m^{\prime}}}}{\partial n}\right) \mathrm{d} s,
$$

where $a(\cdot, \cdot)$ is given in (19). Note that the minus sign before the integral is due to our choice of the unit normal vector $n$ on $\partial B_{R}$ directed into the exterior of $B_{R}$, provided we make the opposite choice on $\partial B_{R^{\prime}}$, so that $n$ is directed into the interior of $B_{R, R}$ on both circles $\partial B_{R}$ and $\partial B_{R^{\prime}}$.

We already know that the $u_{m}$ are orthogonal in $L^{2}\left(B_{R, R^{\prime}}\right)$. Using again the orthogonality of the $\xi_{m}$ in $L^{2}\left(S^{1}\right)$, we see that the last integral also vanishes if $m^{\prime} \neq m$. So the $u_{m}$ are also orthogonal in the sense of the sesquilinear form $a(\cdot, \cdot)$. Hence, by virtue of Lemma 14, to prove the convergence in $H^{2}\left(B_{R, R^{\prime}}\right)$, we simply have to verify that the series with general term $a\left(u_{m}, u_{m}\right)$ converges. By (33), this amounts to showing that

$$
\left|\int_{\partial B_{R} \cup \partial B_{R^{\prime}}}\left(N u_{m} \overline{u_{m}}+M u_{m} \frac{\partial \overline{u_{m}}}{\partial n}\right) \mathrm{d} s\right| \lesssim|m|^{3}\left|f_{m}\right|^{2}+|m|\left|g_{m}\right|^{2}
$$

for large $|m|$. From Proposition 4, the integral on $\partial B_{R^{\prime}}$ does not raise any difficulty. To study the integral on $\partial B_{R}$, let us compute $N u_{m}$ and $M u_{m}$ on $\partial B_{R}$ by using the expressions (21) in polar coordinates. To compute $\Delta u_{m}$, we use the fact that the functions $(r, \theta) \mapsto H_{m}^{(1)}(k r) \xi_{m}(\theta)$ and $(r, \theta) \mapsto H_{m}^{(1)}(i k r) \xi_{m}(\theta)$ belong to the kernel of operators $\Delta+k^{2}$ and $\Delta-k^{2}$, respectively. From the expressions (30) and (31) of $F_{m}$ and $G_{m}$, we easily obtain

$$
\left(\begin{array}{l}
N u_{m}(\theta) \\
M u_{m}(\theta)
\end{array}\right)=\xi_{m}(\theta) \mathbb{T}_{m}\left(\begin{array}{l}
f_{m} \\
g_{m}
\end{array}\right),
$$

where $\mathbb{T}_{m}$ denotes the $2 \times 2$ matrix

$$
\mathbb{T}_{m}:=\left(\begin{array}{cc}
-\frac{m^{2}(1-\nu)}{R^{3}}-2 k^{2} \frac{r_{m} s_{m}}{r_{m}-s_{m}} & \frac{m^{2}(1-\nu)}{R^{2}}+k^{2} \frac{r_{m}+s_{m}}{r_{m}-s_{m}} \\
\frac{m^{2}(1-\nu)}{R^{2}}+k^{2} \frac{r_{m}+s_{m}}{r_{m}-s_{m}} & -\frac{1-\nu}{R}-2 k^{2} \frac{1}{r_{m}-s_{m}}
\end{array}\right) .
$$

Then

$$
\int_{\partial B_{R}}\left(N u_{m} \overline{u_{m}}+M u_{m} \frac{\partial \overline{u_{m}}}{\partial n}\right) \mathrm{d} s=R\left(\begin{array}{l}
f_{m} \\
g_{m}
\end{array}\right)^{*} \mathbb{T}_{m}\left(\begin{array}{c}
f_{m} \\
g_{m}
\end{array}\right)
$$


where the exponent $*$ stands for the conjugate transpose of a matrix. Using (36), we see that

$$
\mathbb{T}_{m}=\left(\begin{array}{cc}
-\frac{2|m|^{3}}{R^{3}}+\mathscr{O}\left(|m|^{2}\right) & -\frac{|m|^{2}(1+\nu)}{R^{2}}+\mathscr{O}(|m|) \\
-\frac{|m|^{2}(1+\nu)}{R^{2}}+\mathscr{O}(|m|) & -\frac{2|m|}{R}+\mathscr{O}(1)
\end{array}\right)
$$

as $|m| \rightarrow+\infty$, which shows that for large $|m|$,

$$
\left|\left(\begin{array}{c}
f_{m} \\
g_{m}
\end{array}\right)^{*} \mathbb{T}_{m}\left(\begin{array}{c}
f_{m} \\
g_{m}
\end{array}\right)\right| \lesssim|m|^{3}\left|f_{m}\right|^{2}+|m|\left|g_{m}\right|^{2},
$$

and (37) follows.

\section{$5 \quad$ Weak formulation in a bounded domain}

From now on, we denote by $\mathcal{S}$ the operator from $H^{3 / 2}\left(\partial B_{R}\right) \times H^{1 / 2}\left(\partial B_{R}\right)$ to $H_{\text {loc }}^{2}\left(\mathbb{R}^{2} \backslash \overline{B_{R}}\right)$ which maps the pair $(f, g)$ of Dirichlet data to the solution $u$ of the Dirichlet problem (27). In this section, we show that our initial problem (1) is equivalent to a problem set in a bounded domain with the help of a Dirichlet-to-Neumann operator $\mathbb{T}$ defined from $H^{3 / 2}\left(\partial B_{R}\right) \times H^{1 / 2}\left(\partial B_{R}\right)$ to $H^{-3 / 2}\left(\partial B_{R}\right) \times H^{-1 / 2}\left(\partial B_{R}\right)$ by

$$
\mathbb{T}\left(\begin{array}{l}
f \\
g
\end{array}\right):=\left(\begin{array}{l}
N u \\
M u
\end{array}\right) \quad \text { where } \quad u:=\mathcal{S}\left(\begin{array}{l}
f \\
g
\end{array}\right) .
$$

As in the preceding section, the definitions of $M$ and $N$ are based here on the unit normal $n$ oriented outside $B_{R}$. That $\mathbb{T}$ is a continuous operator is a direct consequence of Lemma 5. From the proof of Proposition 11, an explicit expression of the operator $\mathbb{T}$ is given by

$$
\mathbb{T}\left(\begin{array}{l}
f \\
g
\end{array}\right)=\sum_{m \in \mathbb{Z}} \mathbb{T}_{m}\left(\begin{array}{l}
f_{m} \\
g_{m}
\end{array}\right) \xi_{m},
$$

where $\mathbb{T}_{m}$ is defined in (38) and $f_{m}$ and $g_{m}$ are the Fourier coefficient of $f$ and $g$ given by (28).

Let us now consider the following problem in the bounded domain $\Omega_{R}:=\Omega \cap B_{R}$, where $R>0$ is sufficiently large so that $B_{R}$ contains the obstacle $\mathcal{D}$ :

$$
\begin{cases}\text { Find } u^{\mathrm{s}} \in H^{2}\left(\Omega_{R}\right) \text { such that } & \\
\Delta^{2} u^{\mathrm{s}}-k^{4} u^{\mathrm{s}}=0 & \text { in } \Omega_{R} \\
\left(\mathcal{B}_{1}\left(u^{\mathrm{s}}+u^{\mathrm{i}}\right), \mathcal{B}_{2}\left(u^{\mathrm{s}}+u^{\mathrm{i}}\right)\right)=(0,0) & \text { on } \partial \Omega \\
\left(\begin{array}{l}
N u^{\mathrm{s}} \\
M u^{\mathrm{s}}
\end{array}\right)=\mathbb{T}\left(\begin{array}{c}
\left.u^{\mathrm{s}}\right|_{\partial B_{R}} \\
\left.\left(\partial u^{\mathrm{s}} / \partial n\right)\right|_{\partial B_{R}}
\end{array}\right) & \text { on } \partial B_{R}\end{cases}
$$

Problems (1) and (41) are equivalent in the sense of the following proposition.

Proposition 12. Assume that problem (1) has a solution $v^{\mathrm{s}}$ in $\Omega$. Then its restriction $\left.v^{\mathrm{s}}\right|_{\Omega_{R}}$ is a solution to problem (41). Conversely, if $u^{\mathrm{s}}$ is a solution to problem (41), then the function $v^{\mathrm{s}}$ which is equal to $u^{\mathrm{s}}$ in $\Omega_{R}$ and $\mathcal{S}\left(\left.u^{\mathrm{s}}\right|_{\partial B_{R}},\left.\left(\partial u^{\mathrm{s}} / \partial n\right)\right|_{\partial B_{R}}\right)$ in $\mathbb{R}^{2} \backslash \overline{B_{R}}$ is a solution to problem (1) in $\Omega$.

Proof. Assume that (1) has a solution $v^{\mathrm{s}}$ in $\Omega$. It is clear that its restriction to $\mathbb{R}^{2} \backslash \overline{B_{R}}$ coincides with $\mathcal{S}\left(\left.v^{\mathrm{s}}\right|_{\partial B_{R}},\left.\left(\partial v^{\mathrm{s}} / \partial n\right)\right|_{\partial B_{R}}\right)$, so that $\mathbb{T}\left(\left.v^{\mathrm{s}}\right|_{\partial B_{R}},\left.\left(\partial v^{\mathrm{s}} / \partial n\right)\right|_{\partial B_{R}}\right)=\left(N v^{\mathrm{s}}, M v^{\mathrm{s}}\right)$. We obtain that $\left.v^{\mathrm{s}}\right|_{\Omega_{R}}$ satisfies problem (41).

Conversely, assume that (41) has a solution $u^{\mathrm{s}}$ in $\Omega_{R}$. We consider the function $v^{\mathrm{s}}$ in $\Omega$ such that $\left.v^{\mathrm{s}}\right|_{\Omega_{R}}=u^{\mathrm{s}}$ and $\left.v^{\mathrm{s}}\right|_{\mathbb{R}^{2} \backslash \overline{B_{R}}}=w^{\mathrm{s}}:=\mathcal{S}\left(\left.u^{\mathrm{s}}\right|_{\partial B_{R}},\left.\left(\partial u^{\mathrm{s}} / \partial n\right)\right|_{\partial B_{R}}\right)$. To prove that $v^{\mathrm{s}}$ is a solution to (1), we just have to verify that it satisfies $\Delta^{2} v^{\mathrm{s}}-k^{4} v^{\mathrm{s}}=0$ in $\Omega$. By construction, it satisfies this equation separately in $\Omega_{R}$ ans $\mathbb{R}^{2} \backslash \overline{B_{R}}$. As $\left(N u^{\mathrm{s}}, M u^{\mathrm{s}}\right)^{\top}=\mathbb{T}\left(\left.u^{\mathrm{s}}\right|_{\partial B_{R}},\left.\left(\partial u^{\mathrm{s}} / \partial n\right)\right|_{\partial B_{R}}\right)^{\top}$, we know that the respective traces, normal derivatives, bending moments and transverse forces of $u^{\mathrm{s}}$ and $w^{\mathrm{s}}$ coincide on $\partial B_{R}$. As shown 
below, this yields the result. Indeed, consider a function $\varphi$ in $C_{0}^{\infty}(\Omega)$, which is the set of infinitely smooth and compactly supported functions in $\Omega$. We have, considering distribution brackets,

$$
\begin{aligned}
\left\langle\Delta^{2} v^{\mathrm{s}}-k^{4} v^{\mathrm{s}}, \varphi\right\rangle & =\left\langle v^{\mathrm{s}}, \Delta^{2} \varphi-k^{4} \varphi\right\rangle \\
& =\int_{\Omega_{R}} u^{\mathrm{s}}\left(\Delta^{2} \varphi-k^{4} \varphi\right) \mathrm{d} x+\int_{\mathbb{R}^{2} \backslash \overline{B_{R}}} w^{\mathrm{s}}\left(\Delta^{2} \varphi-k^{4} \varphi\right) \mathrm{d} x .
\end{aligned}
$$

Using twice Green's formula of Lemma 5 in $\Omega_{R}$ yields

$$
\begin{aligned}
\int_{\Omega_{R}} u^{\mathrm{s}}\left(\Delta^{2} \varphi\right. & \left.-k^{4} \varphi\right) \mathrm{d} x=\int_{\Omega_{R}}\left(\Delta^{2} u^{\mathrm{s}}-k^{4} u^{\mathrm{s}}\right) \varphi \mathrm{d} x \\
& -\int_{\partial B_{R}}\left(\frac{\partial u^{\mathrm{s}}}{\partial n} M \varphi+u^{\mathrm{s}} N \varphi\right) \mathrm{d} s+\int_{\partial B_{R}}\left(\frac{\partial \varphi}{\partial n} M u^{\mathrm{s}}+\varphi N u^{\mathrm{s}}\right) \mathrm{d} s
\end{aligned}
$$

where the integral on $\Omega_{R}$ in the right-hand side vanishes, since $\Delta^{2} u^{\mathrm{s}}-k^{4} u^{\mathrm{s}}=0$ in $\Omega_{R}$. By proceeding in the same way in $\mathbb{R}^{2} \backslash \overline{B_{R}}$, we obtain

$$
\begin{aligned}
\left\langle\Delta^{2} v^{\mathrm{s}}-k^{4} v^{\mathrm{s}}, \varphi\right\rangle= & \int_{\partial B_{R}}\left(\left\{\frac{\partial w^{\mathrm{s}}}{\partial n}-\frac{\partial u^{\mathrm{s}}}{\partial n}\right\} M \varphi+\left\{w^{\mathrm{s}}-u^{\mathrm{s}}\right\} N \varphi\right) \mathrm{d} s \\
& -\int_{\partial B_{R}}\left(\frac{\partial \varphi}{\partial n}\left\{M w^{\mathrm{s}}-M u^{\mathrm{s}}\right\}+\varphi\left\{N w^{\mathrm{s}}-N u^{\mathrm{s}}\right\}\right) \mathrm{d} s .
\end{aligned}
$$

As mentioned above, the four terms between braces vanish. We conclude that $\left\langle\Delta^{2} v^{\mathrm{s}}-k^{4} v^{\mathrm{s}}, \varphi\right\rangle=0$ for all $\varphi \in C_{0}^{\infty}(\Omega)$, which is the desired result.

Let us find now a weak formulation of problem (41). Such a formulation depends on the choice of the boundary conditions only by the associated functional space. That is why we introduce four Hilbert space $\mathcal{H}_{\beta}$, for $\beta=1$ to 4 , associated respectively to the four possible pairs of boundary conditions:

$$
\begin{array}{ll}
\mathcal{H}_{1}:=\left\{u \in H^{2}\left(\Omega_{R}\right),\left.u\right|_{\partial \Omega}=\left.(\partial u / \partial n)\right|_{\partial \Omega}=0\right\} & \text { (clamped plate), } \\
\mathcal{H}_{2}:=\left\{u \in H^{2}\left(\Omega_{R}\right),\left.u\right|_{\partial \Omega}=0\right\} & \text { (simply supported plate), } \\
\mathcal{H}_{3}:=\left\{u \in H^{2}\left(\Omega_{R}\right),\left.(\partial u / \partial n)\right|_{\partial \Omega}=0\right\} & \text { (roller supported plate), } \\
\mathcal{H}_{4}:=H^{2}\left(\Omega_{R}\right) & \text { (free plate). }
\end{array}
$$

All of them can be equipped with the inner product of $H^{2}\left(\Omega_{R}\right)$. Instead of the scattered field $u^{\mathrm{s}}$, it is more convenient to consider the total field $u:=u^{\mathrm{s}}+u^{\mathrm{i}}$ in the weak formulation, where we recall that the incident field $u^{\mathrm{i}}$ satisfies $\Delta^{2} u^{\mathrm{i}}-k^{4} u^{\mathrm{i}}$ in $\mathbb{R}^{2}$. It is then easy to see that (41) is equivalent to

$$
\left\{\begin{array}{l}
\text { Find } u \in \mathcal{H}_{\beta} \text { such that } \\
b(u, v)=\ell(v) \text { for all } v \in \mathcal{H}_{\beta},
\end{array}\right.
$$

where $b$ is the sesquilinear form defined by

$$
b(u, v):=a(u, v)-k^{4} \int_{\Omega_{R}} u \bar{v} \mathrm{~d} x-t(u, v),
$$

$a$ is given by (19) with $\mathcal{O}=\Omega_{R}, t$ is defined by

$$
t(u, v):=\int_{\partial B_{R}}\left(\begin{array}{c}
v \\
\partial v / \partial n
\end{array}\right)^{*} \mathbb{T}\left(\begin{array}{c}
u \\
\partial u / \partial n
\end{array}\right) \mathrm{d} s
$$

and the antilinear form $\ell$ is defined on $\mathcal{H}_{\beta}$ by

$$
\ell(v):=\int_{\partial B_{R}}\left(\begin{array}{c}
v \\
\partial v / \partial n
\end{array}\right)^{*}\left\{\left(\begin{array}{l}
N u^{\mathrm{i}} \\
M u^{\mathrm{i}}
\end{array}\right)-\mathbb{T}\left(\begin{array}{c}
u^{\mathrm{i}} \\
\partial u^{\mathrm{i}} / \partial n
\end{array}\right)\right\} \mathrm{d} s .
$$

Let us notice that from (40), the sesquilinear form $t$ can be expressed as

$$
t(u, v)=\frac{1}{R} \sum_{m \in \mathbb{Z}}\left(\begin{array}{c}
\left(v, \xi_{m}\right)_{L^{2}\left(\partial B_{R}\right)} \\
\left(\partial v / \partial n, \xi_{m}\right)_{L^{2}\left(\partial B_{R}\right)}
\end{array}\right)^{*} \mathbb{T}_{m}\left(\begin{array}{c}
\left(u, \xi_{m}\right)_{L^{2}\left(\partial B_{R}\right)} \\
\left(\partial u / \partial n, \xi_{m}\right)_{L^{2}\left(\partial B_{R}\right)}
\end{array}\right) .
$$




\section{$6 \quad$ Fredholmness}

The weak formulation (42) is equivalent to the equation $B u=L$ in $\mathcal{H}_{\beta}$, where the operator $B: \mathcal{H}_{\beta} \rightarrow \mathcal{H}_{\beta}$ and the element $L \in \mathcal{H}_{\beta}$ are defined from the sesquilinear form $b$ and the antilinear form $\ell$, respectively, with the help of the Riesz Theorem:

$$
\begin{aligned}
(B u, v)_{H^{2}\left(\Omega_{R}\right)} & :=b(u, v) \quad \text { for all } u, v \in \mathcal{H}_{\beta}, \\
(L, v)_{H^{2}\left(\Omega_{R}\right)} & :=\ell(v) \quad \text { for all } v \in \mathcal{H}_{\beta} .
\end{aligned}
$$

Theorem 13. The operator $B$ is a Fredholm operator of index 0 .

To prove this theorem we need two lemmas. The first one is proved in [2].

Lemma 14. For $\nu \in[0,1)$, there exist two constants $c_{0}>0$ and $\lambda_{0} \geq 0$ such that the sesquilinear form a given by (19) satisfies the Gärding inequality

$$
a(u, u) \geq c_{0}\|u\|_{H^{2}(\mathcal{O})}^{2}-\lambda_{0}\|u\|_{L^{2}(\mathcal{O})}^{2} \quad \text { for all } u \in H^{2}(\mathcal{O}) .
$$

Remark 15. By using a similar proof as in [3, Lemma 4.2], we point out that if we restrict ourselves to $u \in \mathcal{H}_{\beta}, \beta=1$ or 2 , then $\lambda_{0}$ can be set to 0 in Lemma 14 .

The second lemma concerns the family of $2 \times 2$ matrices $\mathbb{T}_{m}$ defined in (38) and involved in the expression (43) of the sesquilinear form $t$.

Lemma 16. There exists $m_{0} \in \mathbb{N}$, such that for all $|m|>m_{0}$, we have

$$
-\operatorname{Re}\left\{\left(\begin{array}{l}
f_{m} \\
g_{m}
\end{array}\right)^{*} \mathbb{T}_{m}\left(\begin{array}{c}
f_{m} \\
g_{m}
\end{array}\right)\right\} \geq 0 \quad \text { for all }\left(f_{m}, g_{m}\right) \in \mathbb{C}^{2} .
$$

Proof. The asymptotic behavior (39) of $\mathbb{T}_{m}$ for large $|m|$ shows that

$$
-\operatorname{Re}\left\{\left(\begin{array}{l}
f_{m} \\
g_{m}
\end{array}\right)^{*} \mathbb{T}_{m}\left(\begin{array}{l}
f_{m} \\
g_{m}
\end{array}\right)\right\}=R_{m}^{\text {asy }}\left(f_{m}, g_{m}\right)+R_{m}^{\mathrm{rem}}\left(f_{m}, g_{m}\right),
$$

where

$$
\begin{aligned}
& R_{m}^{\text {asy }}\left(f_{m}, g_{m}\right):=\frac{2|m|^{3}}{R^{3}}\left|f_{m}\right|^{2}+\frac{2|m|^{2}(1+\nu)}{R^{2}} \operatorname{Re}\left(f_{m} \overline{g_{m}}\right)+\frac{2|m|}{R}\left|g_{m}\right|^{2}, \\
& R_{m}^{\mathrm{rem}}\left(f_{m}, g_{m}\right):=\mathscr{O}\left(|m|^{2}\right)\left|f_{m}\right|^{2}+\mathscr{O}(|m|) \operatorname{Re}\left(f_{m} \overline{g_{m}}\right)+\mathscr{O}(1)\left|g_{m}\right|^{2} .
\end{aligned}
$$

We notice on the one hand that

$$
\begin{aligned}
R_{m}^{\text {asy }}\left(f_{m}, g_{m}\right) & \geq \frac{2|m|^{3}}{R^{3}}\left|f_{m}\right|^{2}-\frac{|m|^{3}(1+\nu)}{R^{3}}\left|f_{m}\right|^{2}-\frac{|m|(1+\nu)}{R}\left|g_{m}\right|^{2}+\frac{2|m|}{R}\left|g_{m}\right|^{2} \\
& =(1-\nu)\left(\frac{|m|^{3}}{R^{3}}\left|f_{m}\right|^{2}+\frac{|m|}{R}\left|g_{m}\right|^{2}\right)
\end{aligned}
$$

and on the other hand, that

$$
R_{m}^{\mathrm{rem}}\left(f_{m}, g_{m}\right)=\mathscr{O}\left(|m|^{2}\left|f_{m}\right|^{2}+\left|g_{m}\right|^{2}\right)=\mathscr{O}\left(|m|^{-1}\right) R_{m}^{\text {asy }}\left(f_{m}, g_{m}\right),
$$

which yields the result.

Proof of Theorem 13. Let us choose $m_{0}$ as in Lemma 16 and define the two sesquilinear form $t_{0}$ and $t_{\infty}$ deduced from the expression (43) of $t$ by splitting the series in two parts:

$$
t_{0}(u, v):=\frac{1}{R} \sum_{|m| \leq m_{0}} \ldots \quad \text { and } \quad t_{\infty}(u, v):=\frac{1}{R} \sum_{|m|>m_{0}} \ldots .
$$


Then we decompose the sesquilinear form $b$ into $b=c+d$ where

$$
\begin{aligned}
& c(u, v):=a(u, v)+\lambda_{0} \int_{\Omega_{R}} u \bar{v} \mathrm{~d} x-t_{\infty}(u, v) \quad \text { and } \\
& d(u, v):=-\left(\lambda_{0}+k^{4}\right) \int_{\Omega_{R}} u \bar{v} \mathrm{~d} x-t_{0}(u, v) .
\end{aligned}
$$

On the one hand, we observe that for all $u \in \mathcal{H}_{\beta}$,

$$
\operatorname{Re}\{c(u, u)\}=a(u, u)+\lambda_{0} \int_{\Omega_{R}}|u|^{2} \mathrm{~d} x-\operatorname{Re}\left\{t_{\infty}(u, u)\right\} \geq c_{0}\|u\|_{H^{2}\left(\Omega_{R}\right)}^{2},
$$

where we have used Lemmas 14 and 16. This implies that the operator $C$ which is associated with the sesquilinear form $c$ via Riesz theorem is invertible. On the other hand, the operator $D$ which is associated with the sesquilinear form $d$ via Riesz theorem is compact. This follows from the compactness of the embedding of $H^{2}\left(\Omega_{R}\right)$ in $L^{2}\left(\Omega_{R}\right)$ and the fact that the operator associated with the form $t_{0}$ is a finite sum of operators of rank 2 . We conclude that $B=C+D$ is Fredholm of index 0 .

We are now able to prove the main Theorem 1.

Proof of Theorem 1. From Theorem 13 and the Fredholm theory we obtain that existence is equivalent to uniqueness in problem (41). Uniqueness is given by Theorems 7 and 8 .

Remark 17. In the case of the free plate, an alternative method of ours would have been to use the Analytic Fredholm theorem directly on problem (42) by using analyticity with respect to $k$. The main difficulty is to prove the analytic dependence of the Dirichlet-to-Neumann operator $\mathbb{T}$ with respect to $k$.

\section{References}

[1] M. Abramowitz and I. A. Stegun. Handbook of Mathematical Functions with Formulas, Graphs, and Mathematical Tables. Dover, New York, 1970.

[2] S. Agmon. Lectures on elliptic boundary value problems. Van Nostrand, Princeton, 1965.

[3] L. Bourgeois, L. Chesnel, and S. Fliss. On well-posedness of time-harmonic problems in an unbounded strip for a thin plate model. Commun. Math. Sci., to appear.

[4] L. Bourgeois and A. Recoquillay. The linear sampling method for Kirchhoff-Love infinite plates. submitted.

[5] H. Brezis. Analyse fonctionnelle. Masson, Paris, 1983.

[6] F. Cakoni and D. Colton. A qualitative approach to inverse scattering theory. Applied Mathematical Sciences 188. Springer, New York, 2014.

[7] D. Colton and R. Kress. Inverse Acoustic and Electromagnetic Scattering Theory, 2nd edition. Applied Mathematical Science 93. Springer-Verlag, Berlin, 1989.

[8] M. Farhat, S. Guenneau, and S. Enoch. Finite elements modelling of scattering problems for flexural waves in thin plates: application to elliptic invisibility cloaks, rotators and the mirage effect. $J$. Comput. Phys., 230(6):2237-2245, 2011.

[9] K. Förster and T. Weidl. Trapped modes in an elastic plate with a hole. Algebra i Analiz, 23(1):255$288,2011$.

[10] C. Hazard and M. Lenoir. Determination of scattering frequencies for an elastic floating body. SIAM J. Math. Anal., 24(6):1458-1514, 1993.

[11] G. C. Hsiao and W. L. Wendland. Boundary integral equations. Applied Mathematical Sciences 164. Springer-Verlag, Berlin, 2008. 
[12] R. Kress. Linear Integral Equations. Applied Mathematical Science 82. Springer-Verlag, Berlin, 1989.

[13] J. Lagnese and J.-L. Lions. Modelling analysis and control of thin plates. Recherches en Mathématiques Appliquées 6. Masson, Paris, 1988.

[14] A. N. Norris and C. Vemula. Scattering of flexural waves on thin plates. J. Sound Vib., 181(1):115$125,1995$.

[15] F. W. J. Olver. Asymptotics and Special Functions. Academic Press, New York, 1974.

[16] L. Porter. Trapped waves in thin elastic plates. Wave Motion, 45(1):3-15, 2007.

[17] M. J. A Smith, M. H. Meylan, and R. C. McPhedran. Scattering by cavities of arbitrary shape in an infinite plate and associated vibration problems. J. Sound Vib., 330:4029-4046, 2011.

[18] S. Steinberg. Meromorphic families of compact operators. Arch. Rational Mech. Anal., 31:372-379, 1968.

[19] T. Tyni and V. Serov. Scattering problems for perturbations of the multidimensional biharmonic operator. Inverse Probl. Imaging, 12(1):205-227, 2018. 